\title{
Simulation and Efficient Measurements of Intensities for Complex Imaging Sequences
}

Jensen, Jørgen Arendt; Rasmussen, Morten Fischer; Stuart, Matthias Bo; Tomov, Borislav Gueorguiev

Published in:

Proceedings of IEEE International Ultrasonics Symposium

Link to article, DOI:

10.1109/ULTSYM.2014.0286

Publication date:

2014

Document Version

Early version, also known as pre-print

Link back to DTU Orbit

Citation (APA):

Jensen, J. A., Rasmussen, M. F., Stuart, M. B., \& Tomov, B. G. (2014). Simulation and Efficient Measurements of Intensities for Complex Imaging Sequences. In Proceedings of IEEE International Ultrasonics Symposium (pp. 1164-1167). IEEE. https://doi.org/10.1109/ULTSYM.2014.0286

\section{General rights}

Copyright and moral rights for the publications made accessible in the public portal are retained by the authors and/or other copyright owners and it is a condition of accessing publications that users recognise and abide by the legal requirements associated with these rights.

- Users may download and print one copy of any publication from the public portal for the purpose of private study or research.

- You may not further distribute the material or use it for any profit-making activity or commercial gain

- You may freely distribute the URL identifying the publication in the public portal 
Paper presented at the IEEE International Ultrasonics Symposium, Chicago, Il., USA, 2014:

\section{Simulation and Efficient Measurements of Intensities for Complex Imaging Sequences}

Jørgen Arendt Jensen, Morten Fischer Rasmussen, Matthias Bo Stuart and Borislav G. Tomov

Center for Fast Ultrasound Imaging, Biomedical Engineering group, Department of Electrical Engineering, Bldg. 349, Technical University of Denmark, DK-2800 Kgs. Lyngby, Denmark 


\title{
Simulation and Efficient Measurements of Intensities for Complex Imaging Sequences
}

\author{
Jørgen Arendt Jensen, Morten Fischer Rasmussen, Matthias Bo Stuart and Borislav G. Tomov \\ Center for Fast Ultrasound Imaging, Department of Electrical Engineering, \\ Technical University of Denmark, DK-2800 Lyngby, Denmark
}

\begin{abstract}
It is investigated how linear simulation can be used to predict both the magnitude of the intensities as well as the placement of the peak values. An ultrasound sequence is defined through the normal setup routines for the experimental SARUS scanner, and Field II is then used automatically on the sequence to simulate both intensity and mechanical index (MI) according to FDA rules. A $3 \mathrm{MHz}$ BK Medical 8820e convex array transducer is used with the SARUS scanner. An Onda HFL-0400 hydrophone and the Onda AIMS III system measures the pressure field for three imaging schemes: a fixed focus, single emission scheme, a duplex vector flow scheme, and finally a vector flow imaging scheme. The hydrophone is connected to a receive channel in SARUS, which automatically measures the emitted pressure for the complete imaging sequence. MI can be predicted with an accuracy of 16.4 to $38 \%$. The accuracy for the intensity is from -17.6 to $9.7 \%$, although the measured fields are highly non-linear (several MPa) and linear simulation is used. Linear simulation can, thus, be used to accurately predict intensity levels for any advanced imaging sequence and is an efficient tool in predicting the energy distribution.
\end{abstract}

\section{INTRODUCTION}

Ultrasound imaging sequences undergoing clinical tests and all commercial scanners must obey the safety standards set by FDA rules [1]. These rules stipulate limits on the intensity, mechanical index, and temperature index. The intensity values have to be measured with a traceable accuracy of at least $30 \%$ and the Mechnical Index (MI) with an uncertainty of $15 \%$ [1, p. 10]. This is often complicated to perform, as modern imaging sequences contain a mixture of emissions for anatomic, flow and motion estimation, and these will have different pulse lengths, apodizations, and focal points. Current measurement equipment entails using hydrophones and oscilloscopes, which are not fully synchronized to the scanner. Only a single emission type and direction can therefore be measured at a time, which necessitates that the imaging sequence is taken apart and the individual emissions measured. This is cumbersome for long sequences and often assumptions on the dominating emissions are made and safety factors incorporated. The drawback of this is the uncertainty from the choices, and that the full intensity range is not used, which adversely affects the penetration depth and accuracy of e.g. velocity estimates. A method for using the scanner as the measurement devise was suggested in [2]. It ensures that the correct imaging sequence is always used automatically, and that all emissions are included. The approach, thus, solves some of the inherent problems, but it must still be ensured that the measurement is conducted at the correct spatial positions. If the measured values are above the limits, the sequence must be adjusted and measured again, which is cumbersome.

Daft et al. [3] therefore suggested using simulation to cut down on measurement time. They used a rather complicated non-linear simulation method. This paper suggests using linear Field II simulations prior to measurements to ease the sequence planning and reduce simulation and measurement time. The hypothesis is that the simulation accuracy can be within the $30 \%$ accuracy allowed by the FDA. The approach relies on an automatic decoding of the imaging sequence and then a calibration phase for the emitted pressure. Three different imaging sequences are simulated for a convex array probe and corresponding measurements are conducted with the experimental scanner SARUS to investigate the accuracy.

\section{Simulation OF EMitTed PRESSURES AND INTENSITIES USING FIELD II}

The first parameter to be caclulated is the temporal average intensity given by

$$
I_{t a}(\vec{r})=\frac{1}{T^{\prime}} \int_{0}^{T^{\prime}} I_{i}(t, \vec{r}) d t,
$$

where $I_{i}(t, \vec{r})$ is the instantaneous intensity

$$
I_{i}(t, \vec{r})=\frac{p^{2}(t, \vec{r})}{\rho c}=\frac{p^{2}(t, \vec{r})}{Z} .
$$

where $Z=\rho c$ is the characteristic acoustic impedance, $\rho$ is the density, $t$ is time, $T^{\prime}$ is the period from pulse to pulse, $\vec{r}$ is the position of the measurement, and $c$ is the speed of sound. The discrete version of the temporal average intensity from the sampled acoustic pressure $p(n, \vec{r})$ is:

$$
I_{t a}=\sum_{n=1}^{N} \frac{p^{2}(n, \vec{r})}{Z} \frac{\Delta T}{T_{p r f}}
$$

where $T_{p r f}$ is the time between pulse emissions, $\Delta T$ is the sampling interval, and $N$ is the number of samples in the response. The peak value of $I_{t a}(\vec{r})$ for any spatial position is the one used in the FDA standard, so both the intensity value and its position must be found.

The second quantity to calculate is the mechanical index (MI):

$$
M I=\frac{\left|\min (p(n, \vec{r})) / 10^{6}\right|}{\sqrt{f_{0} / 10^{6}}}
$$


where $\min (p(n, \vec{r}))$ is the peak negative pressure, and $f_{0}$ is the emitted frequency of the probe.

Both quantities are derived from the emitted pressure, which is simulated as [4], [5]:

$$
p(\vec{r}, t)=\rho \frac{\partial v_{i m p}(t)}{\partial t} * h(\vec{r}, t) * e(t)
$$

where $h(\vec{r}, t)$ is the spatial impulse response, and $\partial v_{i m p}(t) / \partial t$ is the probe impulse response. This is the impulse response from the voltage applied to the surface acceleration, assuming that the transducer element vibrates as a piston. The last term $e(t)$ is the electrical excitation of the probe, which is usually a single cycle sinusoid for B-mode imaging and several cycles for flow imaging. Assuming a linear medium the emitted field from a multi element probe with $N$ elements is

$$
p(\vec{r}, t)=\rho \frac{\partial v_{\text {imp }}(t)}{\partial t} * e(t) * \sum_{i=1}^{N} a_{i} h_{i}\left(\vec{r}, t-\tau_{i}\right) .
$$

Here $a_{i}$ is the apodization or weight on element $i$ and $\tau_{i}$ is the time delay for focusing. Field II employs this model for calculating the emitted pressure field and non-linear propagation is neglected [6], [7]. It is assumed that all elements vibrate as pistons, and that the excitation and impulse responses can be assumed to be the same across elements. This is often a valid assumption for commercial, high quality probes.

\section{INTENSITIES FOR COMPLEX IMAGING SEQUENCES}

A modern imaging sequence consists of hundred up to thousands of emissions for a single frame. All contribute to the intensity and they should be properly weighted with their time duration and instantaneous intensity value. The instantaneous intensity should then be weighted according to their relative time duration in the imaging sequence as

$$
I_{t a}=\frac{1}{M} \sum_{m=1}^{M} \sum_{n=1}^{N} \frac{p_{i}^{2}(n, \vec{r})}{Z} \frac{\Delta T}{T_{p r f}(i)}
$$

where $m$ is the emission number and $M$ is the number of emissions in the complete sequence, $T_{p r f}$ is the time between pulse emissions, $\Delta T$ is the sampling interval, and $N$ is the number of samples in the response. Measuring each emission for all spatial positions is cumbersome, and is often not made due to time restrictions, when a traditional oscilloscope approach is employed. A better method is to measure all emissions for the same spatial position. This can be done by using the scanner as the measurement device [2]. Here the hydrophone is connected to one of the inputs in the scanner, and this makes it easy to measure for all emissions at the same location. Averaging of responses is also easy, as the measurement is fully synchronized with the emission. This approach is described in [2], and is the one used in this paper.

\section{Calibration of the simulation}

The simulation must be calibrated for the probe used by finding its impulse response. This could be found from modeling the probe material as described in [8]. This entails knowing all the material parameters for the probe along with

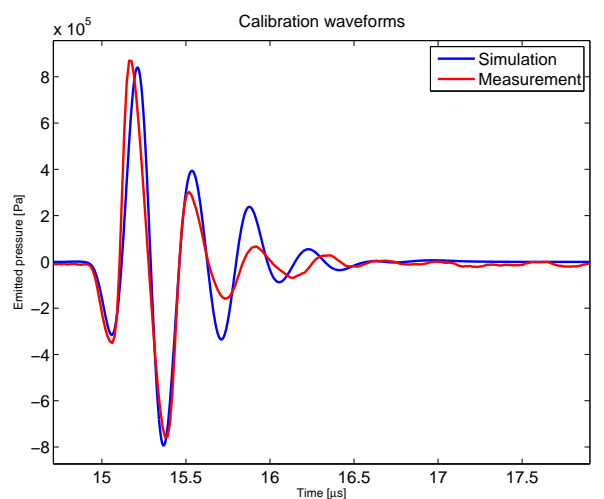

Fig. 1. Waveform used for calibrating the simulation compared to the measured response at a depth of $20 \mathrm{~mm}$ and on the center axis of the transducer. The waveforms have been scaled to have the same energy.

the matching layers. There are often many parameters and layers, and it is often impossible to know all parameters with a sufficient precision, or they are company secrets. The approach taken here is therefore to directly measure the emitted pressure at one position and then use this for calibration. The pressure is then measured by the hydrophone and the response for the exact same setup is simulated with Field II. The impulse response is then amplitude scaled, so the energy of the two responses is the same. This single calibration value is used throughout the simulations. An example of such a calibration waveform is shown in Fig. 1.

\section{Measurement Setup}

The SARUS experimental scanner is used for the experiments [9]. A $3 \mathrm{MHz}$ BK Medical 8820e convex array transducer with $\lambda$ pitch is connected to the SARUS scanner. The transmit impulse response of one transducer element is measured by placing an Onda HFL-0400 hydrophone (Onda Corporation, Sunnyvale, USA) at the elevation focus of the element. The element is excited by a $10 \mu$ s white, Gaussian random signal and the cross-correlation between the excitation and hydrophone signals yields the transmit impulse response. This is used in Field II for simulating the pressure fields from the probe. The Onda AIMS III system measures the pressure field for three imaging schemes: a fixed focus, single emission scheme, a duplex vector flow scheme (Duplex sequence), where a 129 emission B-mode sequence is inter-leaved with flow emissions, and finally a vector flow imaging scheme (VFI sequence) with 17 emission directions of 16 emissions followed by a 129 lines B-mode image.

\section{INTENSITY RESULTS}

Results for the three different sequences are shown in Fig. 2 (single emission), Fig. 3 (VFI) and Fig. 4 (duplex). The top graphs show the derated intensities and the lower derated MI. Measured values are shown on the left, simulated on the right, and the circle indicates the position of the maximum value.

Table I lists the measured and simulated derated MI and $I_{\text {spta. } 3}$ for all three imaging examples. The relative accuracy has also been calculated and spans from 16.4 to $37.9 \%$ for 

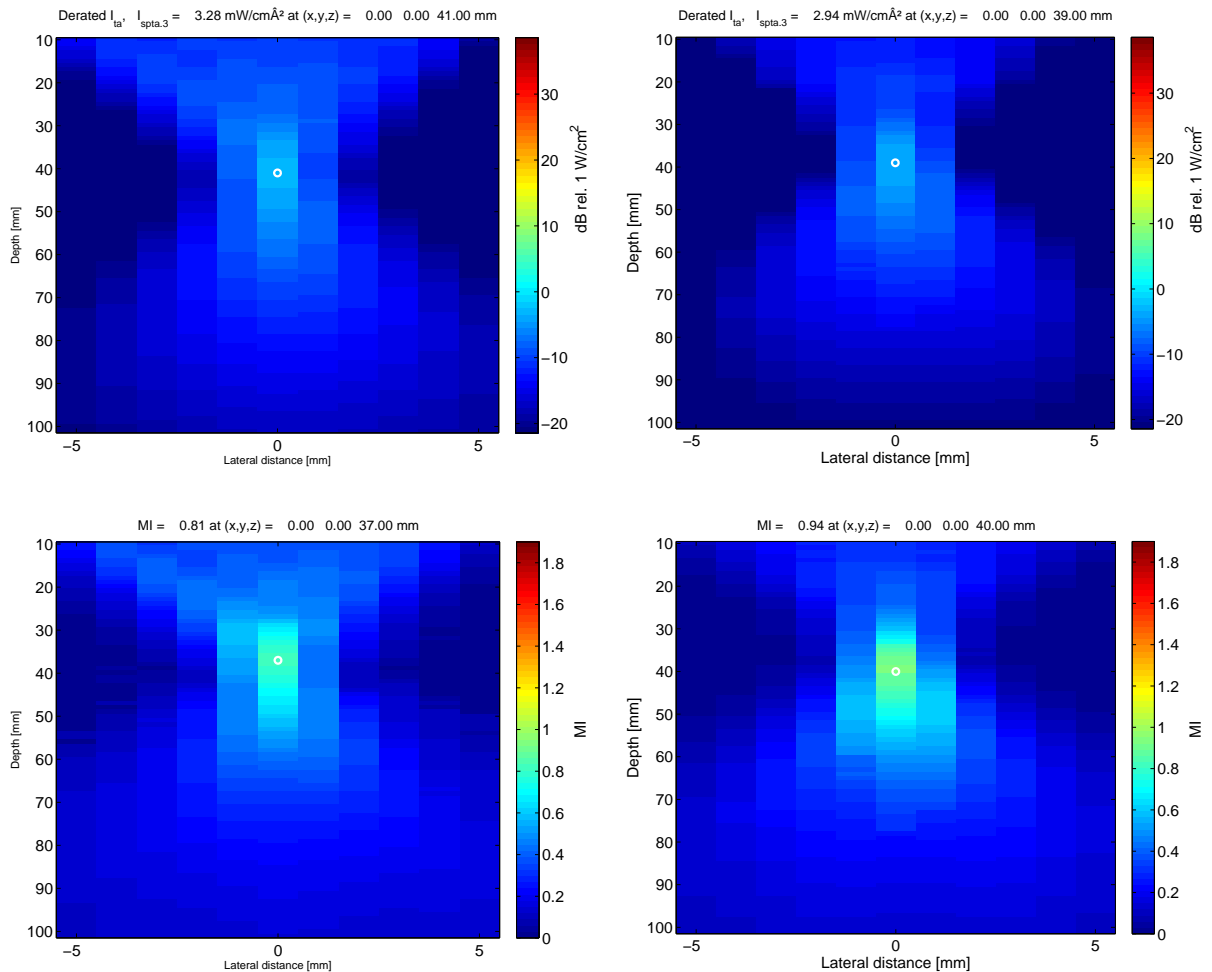

Fig. 2. Results for the single emission sequence as a function of spatial position. The derated temporal averaged intensity is shown in the top graphs and the derated MI is shown on the bottom. The measured distributions are on the left and the simulated on the right. The white circle indicates the position of the peak values.
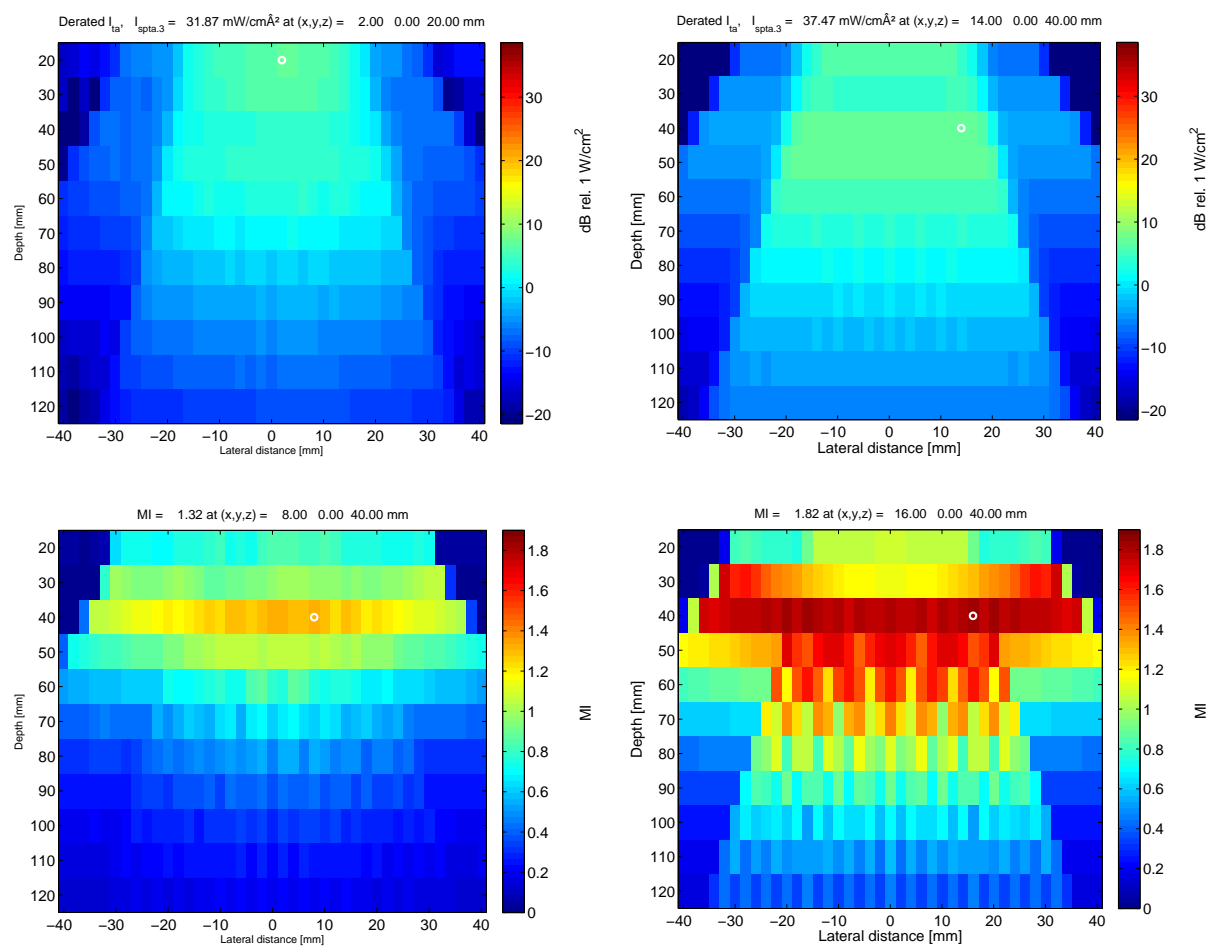

Fig. 3. Results for the vector flow imaging (VFI) sequence as a function of spatial position. The derated temporal averaged intensity is shown in the top graphs and the derated MI is shown on the bottom. The measured distributions are on the left and the simulated on the right. The white circle indicates the position of the peak values. 

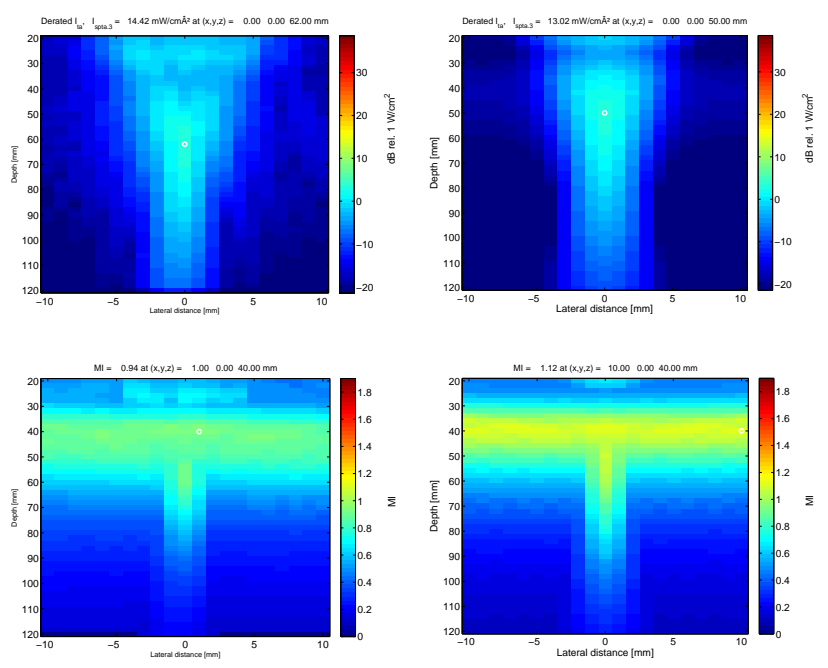

Fig. 4. Results for the duplex B-mode and flow sequence as a function of spatial position. The derated temporal averaged intensity is shown in the top graphs and the derated MI is shown on the bottom. The measured distributions are on the left and the simulated on the right. The white circle indicates the position of the peak values.

TABLE I

MEASURED AND SIMULATED MI AND $I_{\text {spta.3 }}$ FOR ALL THREE IMAGING EXAMPLES ALONG WITH THE RELATIVE ACCURACY.

\begin{tabular}{l|c|r|r|r|c|} 
Imaging sequence & \multicolumn{1}{|c|}{ Para. } & Meas. & Sim. & Rel. acc. & Unit \\
\hline Single emission & MI & 0.81 & 0.94 & $16.4 \%$ & \\
& $I_{\text {spta. } 3}$ & 3.28 & 2.94 & $-8.8 \%$ & $\mathrm{~mW} / \mathrm{cm}^{2}$ \\
\hline Duplex flow & MI & 0.94 & 1.12 & $19.5 \%$ & \\
& $I_{\text {spta.3 }}$ & 14.42 & 13.02 & $9.7 \%$ & $\mathrm{~mW} / \mathrm{cm}^{2}$ \\
\hline VFI & MI & 1.32 & 1.82 & $37.9 \%$ & \\
& $I_{\text {spta.3 }}$ & 31.87 & 37.47 & $-17.6 \%$ & $\mathrm{~mW} / \mathrm{cm}^{2}$ \\
\hline
\end{tabular}

MI. For $I_{\text {spta. } 3}$ the relative accuracy is from $-17.6 \%$ to $9.7 \%$, which is within the required FDA accuracy of $30 \%$. MI is slightly harder to predict due to the influence from non-linear propagation effects, but simulation still can give an early and realistic indication whether a sequence is within the FDA rules.

For the single emission sequence the peak value is predicted to be at $(0,0,39) \mathrm{mm}$, where the intensity measurement gives $(0,0,41) \mathrm{mm}$. For MI it is $(0,0,40) \mathrm{mm}$ and $(0,0,37) \mathrm{mm}$. This is also the easiest and most predictable sequence for the spatial peak values as it has one unique maximum. The VFI sequence has a intensity peak value at $(2,0,20) \mathrm{mm}$, where it is simulated to be at $(14,0,40) \mathrm{mm}$ due to a fairly uniform intensity field in the primary imaging area. The MI position is predicted to be at $(16,0,40) \mathrm{mm}$ and measured to $(8,0,40) \mathrm{mm}$. This is at the B-mode transmit focus that is at the same depth of the different lateral positions. Small variations in the transducer element sensitivity can therefore give a lateral peak position at any of the emitted directions. The same phenomenon is seen for the duplex sequence in Fig. 4, where MI again is dominated by the B-mode focused emission. The intensity is fairly uniform in the central imaging area and has a standard deviation of less than $1.2 \mathrm{~dB}$ in the lateral direction and the largest change is $4.9 \mathrm{~dB}$ from the highest to the lowest value. A $1.2 \mathrm{~dB}$ change is a $15 \%$ change and $4.9 \mathrm{~dB}$ corresponds to a $75 \%$ change in intensity value.

\section{CONCLUSION}

An automatic scheme for simulating intensities for complex ultrasound imaging sequences has been developed. The method is calibrated from a single measurement of the emitted pressure field for a single emission. From this information, and parameters for the scan, it can automatically predict the intensity distribution and MI for any imaging sequence with a mixture of different focal points, apodizations, and excitations. MI can be predicted within an accuracy of 7.5 to $38 \%$. The accuracy for the intensity is from -17.6 to $9.7 \%$, although the measured fields are highly non-linear (several MPa) and the simulations uses the linear simulation program Field II. Simulating the sequences can, thus, give an accurate prediction of the derated values for MI and $I_{\text {spta.3 }}$ nearly within FDA requirements, and this can make the design and scaling of emitted pressure and pulse repetition frequencies much easier and faster for complex imaging sequences.

The simulations cannot replace measurements, but they can give an accurate estimate of the FDA levels and guide where the measurements must be conducted. This can speed up both the measurement process as well as the development cycle for advanced imaging sequences.

\section{ACKNOWLEDGEMENT}

This work was supported by grant 82-2012-4 from the Danish Advanced Technology Foundation and by B-K Medical Aps.

\section{REFERENCES}

[1] FDA, "Information for manufacturers seeking marketing clearance of diagnostic ultrasound systems and transducers," Center for Devices and Radiological Health, United States Food and Drug Administration, Tech. Rep., 2008.

[2] J. A. Jensen, M. F. Rasmussen, M. B. Stuart, and B. G. Tomov, "Rapid measurements of intensities for safety assessment of advanced imaging sequences," in Proc. SPIE Med. Imag., 2014, p. In Press.

[3] C. M. W. Daft, W. M. Leue, K. E. Thomenius, L. A. Odegaard, M. C. MacDonald, and A. S. Meyers, "Acoustic intensity simulations for regulatory compliance," Proceedings of the SPIE - the International Society for Optical Engineering, vol. 3664, pp. 212-220, 1999.

[4] G. E. Tupholme, "Generation of acoustic pulses by baffled plane pistons," Mathematika, vol. 16, pp. 209-224, 1969.

[5] P. R. Stepanishen, "Transient radiation from pistons in an infinite planar baffle," J. Acoust. Soc. Am., vol. 49, pp. 1629-1638, 1971.

[6] J. A. Jensen and N. B. Svendsen, "Calculation of Pressure Fields from Arbitrarily Shaped, Apodized, and Excited Ultrasound Transducers," IEEE Trans. Ultrason., Ferroelec., Freq. Contr., vol. 39, pp. 262-267, 1992.

[7] J. A. Jensen, "Field: A program for simulating ultrasound systems," Med. Biol. Eng. Comp., vol. 10th Nordic-Baltic Conference on Biomedical Imaging, Vol. 4, Supplement 1, Part 1, pp. 351-353, 1996.

[8] D. Bæk, J. A. Jensen, and M. Willatzen, "Modeling transducer impulse responses for predicting calibrated pressure pulses with Field II," $J$. Acoust. Soc. Am., vol. 127, no. 5, pp. 2825-2835, May 2010.

[9] J. A. Jensen, H. Holten-Lund, R. T. Nilsson, M. Hansen, U. D. Larsen, R. P. Domsten, B. G. Tomov, M. B. Stuart, S. I. Nikolov, M. J. Pihl, Y. Du, J. H. Rasmussen, and M. F. Rasmussen, "SARUS: A synthetic aperture real-time ultrasound system," IEEE Trans. Ultrason., Ferroelec., Freq. Contr., vol. 60, no. 9, pp. 1838-1852, 2013. 